Research Article

\title{
A randomized comparison between lercanidipine and amlodipine for efficacy and tolerability in patients with essential hypertension
}

\author{
Girish Tulshidas Raparti ${ }^{1 *}$, Balwant Kisanrao Choure ${ }^{2}$, Praveenkumar Tukaram Patil ${ }^{3}$, \\ Shailesh Shyamling Patne ${ }^{4}$
}

\begin{abstract}
${ }^{1}$ Department of Pharmacology, Ashwini Rural Medical College and Hospital, Solapur,

Maharashtra, India

${ }^{2}$ Department of Pharmacology, RCSM Government Medical College, Kolhapur, Maharashtra, India

${ }^{3}$ Department of Pharmacology, Shri Bhausaheb Hire Medical College, Dhule, Maharashtra, India

${ }^{4}$ Department of Pathology, Ashwini Rural Medical College and Hospital, Solapur,

Maharashtra, India
\end{abstract}

Received: 03 July 2016 Accepted: 08 July 2016

\section{*Correspondence to:}

Dr. Girish Tulshidas Raparti, Email: girishraprti@yahoo.in

Copyright: (C) the author(s), publisher and licensee Medip Academy. This is an openaccess article distributed under the terms of the Creative Commons Attribution NonCommercial License, which permits unrestricted noncommercial use, distribution, and reproduction in any medium, provided the original work is properly cited.

\begin{abstract}
Background: Lercanidipine, a newly added dihydropyridine, was compared with one of its older and time tested congener- amlodipine for their efficacy and tolerability.

Methods: This was a prospective, double blind, parallel group study. 100 patients according to inclusion and exclusion criteria were randomised into two groups of 50 each. One group received lercanidipine $10 \mathrm{mg}$ while second group received tablet amlodipine $5 \mathrm{mg}$ at the beginning, both once daily orally for 12 weeks of duration. Follow up was done at 2, 4, 8 and 12 weeks. Systolic and diastolic blood pressure was recorded in sitting position. If the patient did not attain target blood pressure of $140 / 90 \mathrm{mmHg}$, dose was titrated at 4,8 weeks. Tolerability was assessed by questioning about adverse drug reactions at follow up and derangements of routine laboratory parameters at the end. $\mathrm{Z}$ test was used for analysis.

Results: There was no statistical difference between antihypertensive efficacies of two drugs. Number of patients showing adverse reactions were significantly less in lercanidipine group compared to amlodipine. Though vasodilation related adverse reactions were less lercanidipine group, significant difference was observed only in occurrence of pedal edema. This difference in incidence of edema cannot be related to the extent of reduction in blood pressure.

Conclusions: With the comparable antihypertensive efficacy, lercanidipine is associated with considerably lower incidence of vasodilation related side effects than amlodipine, especially pedal edema. This favourable tolerability profile can potentially enhance treatment outcome by promoting better adherence to drug therapy.
\end{abstract}

Keywords: Lercanidipine, Amlodipine, Hypertension, Edema

\section{INTRODUCTION}

Hypertension is a global health care problem. Since last few decades, prevalence of hypertension has increased in
India, especially in urban population. ${ }^{1}$ It is a wellrecognised risk factor for the cardiovascular diseases. ${ }^{2}$ It occurs commonly with diabetes, which itself is a major cardiovascular risk factor. ${ }^{3}$ 
Calcium channel blocker (CCB) class of drugs comprises three groups of compounds with distinct pharmacodynamics effect. Dihydropyridines group of CCBs are recognised as well-tolerated and safe drugs. They are considered as one of the first line antihypertensive drugs. But the main troublesome adverse reactions of them are the development of pedal edema and other vasodilation related side effects like headache, dizziness, flushing, palpitation etc. ${ }^{4}$ This poor drug tolerability can lead to poor compliance of the therapy. It is stated that one in four patients discontinue antihypertensive treatment within the first year of therapy because of adverse reactions. ${ }^{5-7}$ Moreover, this edema may get worsen with time leading to hyperpigmentation and discoloration of skin. This can lead to dose reduction or prevent use of this effective class of drugs.

Lercanidipine is a derivative of third generation CCBs, claim to have even and sustained blood pressure lowering with once-daily dosing. Common adverse drug reactions related to $\mathrm{CCBs}$ such as pedal edema, headache, dizziness, palpitation etc. are said to be low with this vasoselective dihydropyridine congener. Very few clinical trials have been conducted comparing this drug with one of its older and time tested congeneramlodipine. As tolerability to antihypertensive may vary between populations, this study was undertaken to evaluate the efficacy and tolerability of lercanidipine in patients of essential hypertension attending tertiary care hospital.

\section{METHODS}

This was a prospective, randomized, double blind, parallel group study carried out at a tertiary care hospital over a year after obtaining approval by institutional ethics committee. Newly diagnosed patients of both sexes and age more than 35 years with mild to moderate essential hypertension (systolic blood pressure between 140 and $179 \mathrm{mmHg}$ and diastolic blood pressure between 90 and $109 \mathrm{mmHg}$ ) were enrolled in this study after receiving informed written consent. The following category of patients were excluded: patients on other antihypertensive drugs, secondary hypertension, obstructive biliary disease, cholestasis or hepatic impairment, renal impairment, aortic stenosis, unstable angina, uncontrolled heart failure and MI within 1 month of attack pregnant and lactating women, female patients of child bearing age group not using medically approved contraceptives.

Sample size determined from previous studies was large but for feasibility a smaller sample was chosen. 144 patients attending OPD were screened; out of which 100 patients were enrolled in the study and were randomised into two groups of 50 each (Figure 1). Simple randomization was done and allocation was concealed by employing different investigators for each step of random number generation, enrolment, assignment of patients to treatment groups. Participants and investigators were blinded to achieve double blind. Patients in first group received tablet lercanidipine $10 \mathrm{mg}$ while second group patients received tablet amlodipine $5 \mathrm{mg}$ in the beginning, both once daily orally for 12 weeks of duration. Follow up was done at 2, 4, 8 and 12 weeks. At each visit, patients were clinically examined and medical history was noted. All patients advised lifestyle modifications. At each visit heart rate was noted, systolic and diastolic blood pressure (BP) was recorded in sitting position after 10 minutes of rest by auscultation method using mercury sphygmomanometer. The patients were advised to avoid smoking or drinking coffee within 30 minutes before assessment of BP. Laboratory investigations like serum creatinine, SGOT, SGPT, random blood sugar level were carried out at first day and 12 weeks of study.

The primary efficacy parameters were the reduction in baseline systolic and diastolic BP. If the patient did not attain the target blood pressure of $140 / 90 \mathrm{mmHg}$, the dose was titrated at 4 th and 8 th weeks by $5 \mathrm{mg}$ and $2.5 \mathrm{mg}$ in lercanidipine and amlodipine groups respectively.

Patients who did not attain target BP level at the end of study were labelled as non-responders and referred to physician for further treatment. Tolerability and safety was assessed by presence or absence of adverse drug reactions, and derangement of laboratory parameters. Signs and symptoms namely pedal edema, headache, dizziness, flushing, palpitation, fatigue, constipation, nausea, vomiting, muscle cramps, dyspepsia, difficulty in micturition, day time sleepiness, tachycardia and rash were noted.

Data was checked for normality. Qualitative data was analysed by using $\mathrm{Z}$ test for difference between two proportions or Fisher's exact test for small sample sized data. Quantitative data was analysed using $\mathrm{Z}$ test for difference between two means. $\mathrm{P}$ value $<0.05$ was taken as significant and $\mathrm{p}$ value $<0.001$ was considered as highly significant; while $\mathrm{p}$ value $>0.05$ was regarded as non-significant.

\section{RESULTS}

Baseline values of all three groups were comparable with respect to age, sex, habits, systolic BP, diastolic BP and heart rate (Table 1).

Table 1: Baseline data of lercanidipine and amlodipine groups.

\begin{tabular}{|llll|}
\hline Parameters & $\begin{array}{l}\text { Lercanidipine } \\
\mathbf{n}_{\mathbf{1}}=\mathbf{4 5} \\
(\mathbf{m} \text { ean } \pm \text { SD) }\end{array}$ & $\begin{array}{l}\text { Amlodipine } \\
\mathbf{n}_{\mathbf{2}}=\mathbf{4 4} \\
(\mathbf{m} \text { ean } \pm \text { SD })\end{array}$ & p value \\
\hline $\begin{array}{l}\text { Systolic BP } \\
(\mathrm{mmHg})\end{array}$ & $156.04 \pm 9.52$ & $156.81 \pm 9.42$ & $\mathrm{p}>0.05$ \\
\hline $\begin{array}{l}\text { Diastolic BP } \\
(\mathrm{mmHg})\end{array}$ & $97.15 \pm 4.21$ & $97.5 \pm 4.44$ & $\mathrm{p}>0.05$ \\
\hline $\begin{array}{l}\text { Heart rate } \\
(\mathrm{bpm})\end{array}$ & $75.47 \pm 5.47$ & $75.22 \pm 4.69$ & $\mathrm{p}>0.05$ \\
\hline
\end{tabular}


In both lercanidipine and amlodipine treated groups, the reduction in systolic $\mathrm{BP}$ was found to be highly statistically significant $(p<0.001)$ at $2,4,8$ and 12 weeks of therapy, when compared with the baseline readings
(Table 2). The reduction in diastolic BP was also found to be statistically significant $(\mathrm{p}<0.001)$ at $2,4,8$ and 12 weeks of therapy, when compared with the baseline readings, in both the groups.

Table 2: Effect of drugs on mean systolic and diastolic blood pressure (mmHg) at 2, 4, 8 and 12 weeks.

\begin{tabular}{|c|c|c|c|c|}
\hline \multirow{2}{*}{ Duration } & \multicolumn{2}{|c|}{ Systolic BP (mean \pm SD) } & \multicolumn{2}{|c|}{ Diastolic BP (mean \pm SD) } \\
\hline & Lercanidipine $n_{1}=45$ & Amlodipine $n_{2}=44$ & Lercanidipine $n_{1}=45$ & Amlodipine $n_{2}=44$ \\
\hline Day 0 & $156.04 \pm 9.52$ & $156.81 \pm 9.42$ & $97.15 \pm 4.21$ & $97.5 \pm 4.44$ \\
\hline 2 weeks & $144.04 \pm 6.65$ & $145.86 \pm 7.11$ & $88.97 \pm 3.00$ & $89.40 \pm 3.03$ \\
\hline 4 weeks & $139.64 \pm 6.67$ & $141.02 \pm 6.95$ & $86.35 \pm 2.67$ & $86.95 \pm 2.74$ \\
\hline 8 weeks & $135.26 \pm 5.84$ & $136.68 \pm 6.58$ & $84.71 \pm 3.46$ & $85.13 \pm 3.21$ \\
\hline 12 weeks & $132.4 \pm 5.86$ & $134 \pm 6.51$ & $82.88 \pm 3.37$ & $83.63 \pm 3.43$ \\
\hline
\end{tabular}

Table 3: Comparison of mean reduction in systolic and diastolic blood pressure $(\mathrm{mmHg})$ from the baseline.

\begin{tabular}{|c|c|c|c|c|c|c|}
\hline \multirow{2}{*}{ Duration } & \multicolumn{2}{|c|}{ Systolic BP reduction (mean \pm SD) } & \multirow{2}{*}{ P value } & \multicolumn{2}{|c|}{ Diastolic BP reduction (mean \pm SD) } & \multirow{2}{*}{ P value } \\
\hline & Lercanidipine $n_{1}=45$ & Amlodipine $\mathrm{n}_{1}=44$ & & Lercanidipine $n_{1}=45$ & Amlodipine $\mathrm{n}_{1}=44$ & \\
\hline 2 weeks & $12.00 \pm 3.27$ & $10.95 \pm 3.54$ & $\mathrm{p}>0.05$ & $8.17 \pm 1.52$ & $8.09 \pm 1.92$ & $\mathrm{p}>0.05$ \\
\hline 4 weeks & $16.4 \pm 3.45$ & $15.79 \pm 3.55$ & $\mathrm{p}>0.05$ & $10.8 \pm 2.31$ & $10.54 \pm 2.63$ & $\mathrm{p}>0.05$ \\
\hline 8 weeks & $20.77 \pm 4.27$ & $19.95 \pm+4.81$ & $\mathrm{p}>0.05$ & $12.44 \pm 1.75$ & $12.36 \pm 2.25$ & $\mathrm{p}>0.05$ \\
\hline 12 weeks & $23.6 \pm 4.14$ & $22.81 \pm 4.12$ & $\mathrm{p}>0.05$ & $14.26 \pm 1.98$ & $13.86 \pm 2.04$ & $\mathrm{p}>0.05$ \\
\hline
\end{tabular}

Table 4: Adverse drug reactions observed in both the groups.

\begin{tabular}{|lll|}
\hline Adverse reactions & $\begin{array}{l}\text { Lercanidipine } \\
\mathbf{n}_{\mathbf{1}}=\mathbf{4 5}\end{array}$ & $\begin{array}{l}\text { Amlodipine } \\
\mathbf{n}_{\mathbf{2}}=\mathbf{4 4}\end{array}$ \\
\hline Pedal edema* & 1 & 8 \\
\hline Headache & 2 & 4 \\
\hline Flushing & 1 & 2 \\
\hline Tachycardia & - & 1 \\
\hline Dizziness & - & 1 \\
\hline Fatigue & 1 & 1 \\
\hline Constipation & - & 1 \\
\hline $\begin{array}{l}\text { Total number of } \\
\text { adverse reactions }\end{array}$ & 5 & 18 \\
\hline $\begin{array}{l}\text { Total number of } \\
\text { patients } \\
\text { showing adverse } \\
\text { reactions* }\end{array}$ & 4 & 13 \\
*P Value significant $(<0.05)$. &
\end{tabular}

Table 5: Effect of drugs on laboratory parameters and heart rate.
The mean reduction in systolic BP in lercanidipine group was $12.00 \pm 3.27 \mathrm{mmHg}$ at 2 weeks, $16.4 \pm 3.45 \mathrm{mmHg}$ at 4 weeks, $20.77 \pm 4.27 \mathrm{mmHg}$ at 8 weeks and $23.6 \pm 4.14$ $\mathrm{mmHg}$ at 12 weeks of treatment (Table 3). While the mean reduction in systolic BP in amlodipine group was $10.95 \pm 3.54 \mathrm{mmHg}$ at 2 weeks, $15.79 \pm 3.55 \mathrm{mmHg}$ at 4 weeks, $19.95 \pm 4.81 \mathrm{mmHg}$ at 8 weeks and $22.81 \pm 4.12$ $\mathrm{mmHg}$ at 12 weeks of treatment. When the reduction in systolic BP in two groups was compared, there was no significant difference between the two groups $(\mathrm{p}>0.05)$. The mean reduction in diastolic BP in lercanidipine group was $8.17 \pm 1.52 \mathrm{mmHg}$ at 2 weeks, $10.8 \pm 2.31 \mathrm{mmHg}$ at 4 weeks, $12.44 \pm 1.75 \mathrm{mmHg}$ at 8 weeks and $14.26 \pm 1.98$ $\mathrm{mmHg}$ at 12 weeks. While the mean reduction in diastolic BP in amlodipine group was $8.09 \pm 1.92 \mathrm{mmHg}$ at 2 weeks, $10.54 \pm 2.63 \mathrm{mmHg}$ at 4 weeks, $12.36 \pm 2.25 \mathrm{mmHg}$ at 8 weeks and $13.86 \pm 2.04 \mathrm{mmHg}$ at 12 weeks. When these values were compared between two groups, the difference was not statistically significant $(\mathrm{p}>0.05)$.

\begin{tabular}{|c|c|c|c|c|c|c|}
\hline \multirow{2}{*}{ Parameters } & \multicolumn{2}{|c|}{ Lercanidipine $n_{1}=45($ mean \pm SD) } & \multirow{2}{*}{ p value } & \multicolumn{2}{|c|}{ Amlodipine $n_{1}=44($ mean \pm SD $)$} & \multirow{2}{*}{ p value } \\
\hline & Before treatment & After treatment & & Before treatment & After treatment & \\
\hline Creatinine $(\mathrm{mg} / \mathrm{dl})$ & $0.98 \pm 0.29$ & $0.91 \pm 0.23$ & $\mathrm{p}>0.05$ & $1.04 \pm 0.21$ & $0.95 \pm 0.27$ & $\mathrm{p}>0.05$ \\
\hline SGPT (IU/L) & $21.63 \pm 6.97$ & $21.11 \pm 6.34$ & $\mathrm{p}>0.05$ & $23.29 \pm 5.81$ & $23.92 \pm 5.51$ & $\mathrm{p}>0.05$ \\
\hline SGOT (IU/L) & $23.48 \pm 7.11$ & $24.09 \pm 7.24$ & $p>0.05$ & $25.23 \pm 6.11$ & $25.98 \pm 5.93$ & $\mathrm{p}>0.05$ \\
\hline $\mathrm{BSL}(\mathrm{mg} / \mathrm{dl})$ & $99.42 \pm 8.33$ & $98.42 \pm 8.72$ & $\mathrm{p}>0.05$ & $98.99 \pm 9.94$ & $99.74 \pm 8.99$ & $\mathrm{p}>0.05$ \\
\hline Heart rate $(\mathrm{bpm})$ & $75.47 \pm 5.45$ & $74.94 \pm 3.93$ & $p>0.05$ & $75.22 \pm 4.69$ & $74.65 \pm 3.28$ & $\mathrm{p}>0.05$ \\
\hline
\end{tabular}




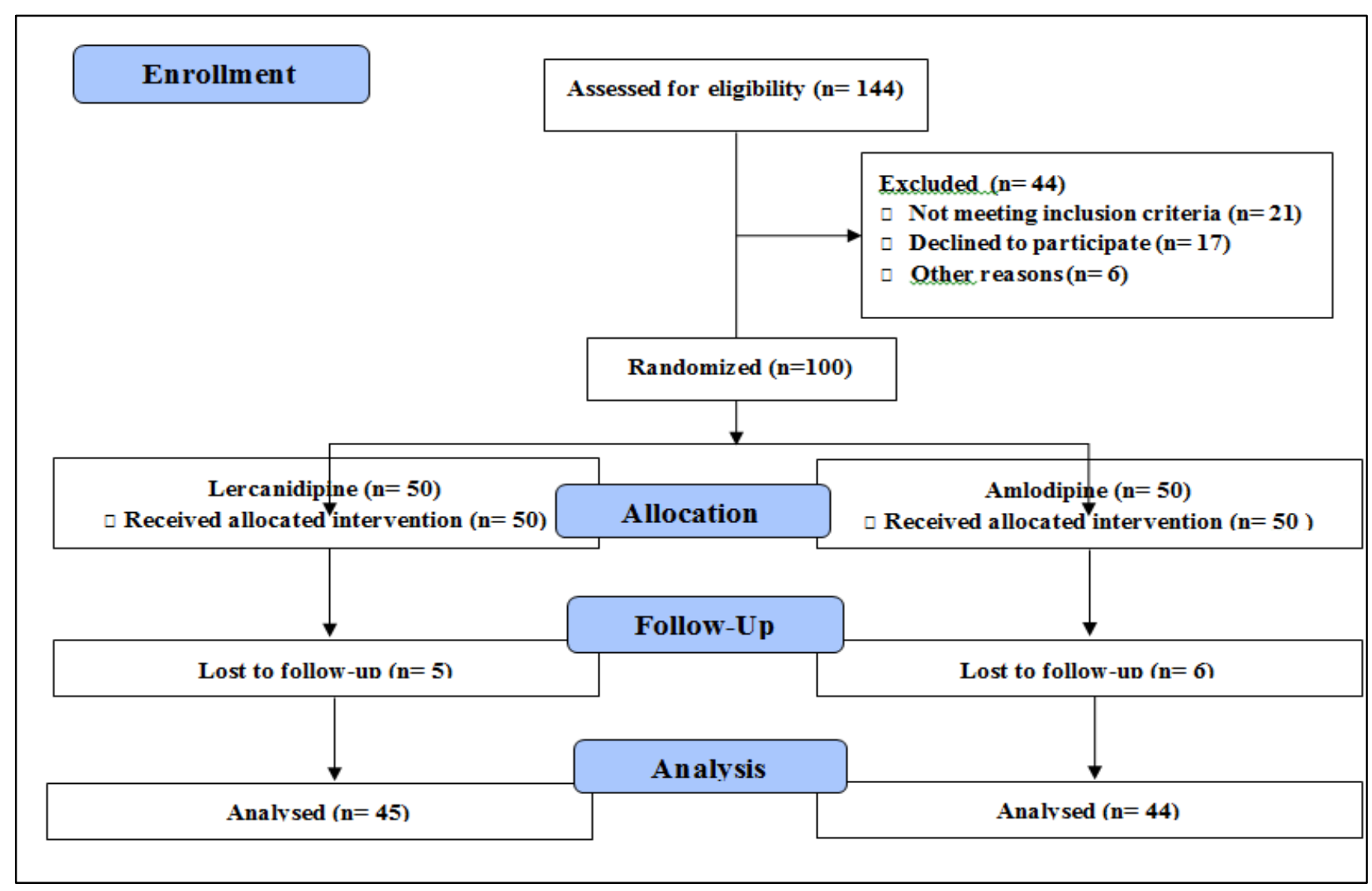

Figure 1: Participant flow.

6 patients in lercanidipine group and 7 patients in amlodipine group not achieved target BP at the end of study. These patients were labelled as non-responders. There was no statistical difference found in number of non-responders between two groups ( $\mathrm{p}>0.05)$.

In lercanidipine treated group, adverse reactions noted were peripheral edema, headache, flushing and fatigue. In addition to these, amlodipine treated patient reported tachycardia, dizziness and constipation. As shown in table 4, 4 patients reported 5 adverse events in lercanidipine treated group as compared to 13 patients showing 18 adverse reactions in amlodipine group. The difference in number of patients reporting adverse reactions between lercanidipine and amlodipine group was found statistically significant $(\mathrm{p}<0.05)$.

3 patients in lercanidipine group experienced 4 vasodilatory adverse reactions (viz. peripheral edema, headache and flushing) while in amlodipine group 11 patients showed 16 vasodilation related side effects (viz. peripheral edema, headache, flushing, dizziness and tachycardia). In lercanidipine group, 1 patient had reported pedal edema while 8 patients had showed pedal edema in amlodipine treated group. When two groups were compared, the incidence of pedal edema was significantly higher in amlodipine group $(\mathrm{p}<0.05)$. There was no significant difference observed in mean blood pressure of patients with or without pedal edema within both the groups $(\mathrm{p}>0.05)$. Though numbers of various adverse effects other than pedal edema were more in amlodipine treated group, when this difference was compared, it was found non-significant $(\mathrm{p}>0.05)$ (Table 4).

Table 5 shows the values of serum creatinine, SGPT, SGOT, random blood sugar level and heart rate at the baseline and at the end of the study in both the groups. There was no significant differences observed in these values $(p>0.05)$ before and after treatment.

\section{DISCUSSION}

Management of hypertension, a major cardiovascular risk factor, practically requires lifelong drug therapy to achieve strict blood pressure control. ${ }^{8}$ To improve compliance of the drug treatment, better tolerated antihypertensive agents are required.

CCBs have been studied for its effect on the cardiovascular safety. Pedal edema is one of the commonly observed side effect with dihydropyridine group of CCBs. Edema is dose dependent, may exceed $80 \%$ with very high doses of dihydropyridines. ${ }^{9}$ Amlodipine is a well-established and commonly prescribed drug in its class. But different tolerability pattern can be seen between compounds of the same class. ${ }^{10}$ Therefore this study was undertaken to compare 
lercanidipine, a newly added dihydropyridine congener, with commonly used dihydropyridine amlodipine.

This study showed that lercanidipine significantly lowered blood pressure within 15 days of the therapy compared to base line in majority of the patients. A consistent increment in the antihypertensive action of lercanidipine was observed throughout study period. When antihypertensive efficacy of lercanidipine was compared with amlodipine, both drugs seem to be equally effective in reducing systolic and diastolic BP. The difference in non-responders between two groups was also statistically insignificant.

Table 5 shows data related with tolerability of the two drugs in the study. 4 patients reported 5 adverse reactions in lercanidipine treated group as compared to 13 patients showing 18 adverse reactions in amlodipine group. This difference in number of patients reporting adverse reactions between two group was statistically significant $(\mathrm{p}<0.05)$.

In the study, patients treated with lercanidipine had experienced lower rates of vasodilatory side effects than those who received amlodipine. Among all vasodilation related side effects observed, major difference in incidence was observed in pedal edema. In lercanidipine group, 1 patient experienced pedal edema while 8 patients reported it in amlodipine treated group. This difference was found to be statistically significant $(\mathrm{p}<0.05)$. Similar reports have been shown in some of the earlier studies. Leonetti et al. has found significantly higher rates of edema in amlodipine treated group compared to lercanidipine. ${ }^{10}$ Observations in another study indicated that for any given fall in blood pressure, the incidence of vasodilatory edema was significantly less with lercanidipine compared with the few second-generation calcium channel blockers including amlodipine. ${ }^{11}$ This difference in incidence of edema cannot be related to extent of reduction in blood pressure, as the magnitude of blood pressure reduction is similar in both the groups and no difference in magnitude of antihypertensive effect was observed in patients with or without edema.

The edema is outcome of capillary fluid filtration into the interstitial space of the tissue. Normally, postural vasoconstriction occurs in both the arteriolar and the venous limb of the blood vessels when there is a change from the supine to the standing position. This venoarteriolar reflex maintains the capillary fluid filtration constant. The precapillary arteriolar vasoconstriction is selectively diminished by CCBs. They appear to block the myogenic component of the reflex control of the cutaneous blood flow, which is independent of neural, metabolic, and other hormonal influences. ${ }^{12}$ This could be responsible for rise in intracapillary pressure, which results in capillary fluid filtration into the interstitium. This leads to formation of edema which seems to be exaggerated by gravity.
Lercanidipine seems to have different set of influence on the blood vessels compared to older CCBs. Experimental studies have shown that lercanidipine also has a distinct vasodilatory effect on the efferent arteriole in addition to the afferent arteriole in the kidney. ${ }^{13}$ Thus, it was stated that lercanidipine provides a more balanced pre- and postglomerular dilation, thereby reducing intracapillary pressure. It was hypothesized that such a balanced vasodilator action could take place in other capillary beds as well, which results in decreased incidence of the edema. $^{11}$

Some studies have proposed other possible mechanisms. One hypothesis suggests that lercanidipine causes lesser venoconstriction than other drugs due to lower sympathetic activation. Fogari et al. studied this difference by estimating serum levels of norepinephrine. It was seen that lercanidipine treated patients showed lesser norepinephrine levels than patients treated with nifedipine GITS. ${ }^{14}$ A different effect on vascular permeability and consequent fluid extravasation has also been suggested. ${ }^{15}$ Another hypothesis states that different pattern of pharmacological action of lercanidipine is responsible for its favourable tolerability profile. Lercanidipine proposed to have a greater solubility within the arterial cellular membrane bilayer compared to other long acting dihydropyridines. This results in longer stay in the blood vessels and consequent long duration of action even though it has relatively short plasma half-life. Therefore it was suggested that rapid removal of lercanidipine from plasma may be responsible for its favourable tolerability profile. $^{16}$

Though incidence of vasodilation related side effects other than pedal edema were less in lercanidipine treated group as compared to amlodipine group, the difference was statistically not significant. This observation was similar to the findings of the ELYPSE and the ELECTRA study. ${ }^{17,18}$

No drug had any adverse impact on the values of serum creatinine, SGPT, SGOT, blood sugar level and heart rate in this study.

Apart from the efficacy parameters studied in the present study, various other favourable effects of lercanidipine have been observed in previous studies. Human studies have demonstrated that lercanidipine is equally effective in young and old patients (especially in isolated systolic hypertension). It is also effective in patients associated with comorbid conditions such as type 2 diabetes and/or renal dysfunction. ${ }^{2}$ It is also stated that its stable blood pressure control without marked hypotension during the night hours, which can be related to coronary events and strokes, will promises cardiovascular safety. ${ }^{19}$

Therefore, lercanidipine appears to be well tolerated in all age groups with favorable efficacy. Findings of the present study and observations from the previous clinical trials make lercanidipine a flexible choice for 
antihypertensive treatment across a broad range of patients.

Despite its advantages, one disadvantage of lercanidipine is its higher cost compared to amlodipine. The present study is a small study both as regards to the number of patients included and the duration. In India more extensive studies including large number of patients with differing severity and comorbidities; considering more efficacy parameters to evaluate long term effect and compliance are required to determine the exact utility of this drug.

Thus it can be concluded that, for the comparable antihypertensive efficacy, lercanidipine is associated with considerably lower incidence of vasodilation related side effects than amlodipine, especially pedal edema. This favorable tolerability profile can potentially enhance treatment outcome by promoting better adherence to drug therapy.

\section{Funding: No funding sources}

Conflict of interest: None declared

Ethical approval: The study was approved by the Institutional Ethics Committee

\section{REFERENCES}

1. Das SK, Sanyal K, Basu A. Study of urban community survey in India: growing trend of high prevalence of hypertension in a developing country. Int J Med Sci. 2005;2(2):70-8.

2. Borghi C. Lercanidipine in hypertension. Vasc Health Risk Manag. 2005;1(3):173-82.

3. Arauz-Pacheco C, Parrott MA, Raskin P. Hypertension management in adults with diabetes. Diabetes Care, 2004;27(Supp11):S65-7.

4. Weir MR. Incidence of pedal edema formation with dihydropyridine calcium channel blockers: issues and practical significance. J Clin Hypertens (Greenwich). 2003;5(5):330-5.

5. Düsing R, Weisser B, Mengden T, Vetter H. Changes in antihypertensive therapy- the role of adverse effects and compliance. Blood Press. 1998;7:313-5.

6. Aranda P, Tamargo J, Aranda FJ, Luque M, LópezGarcia-Franco A. Use and adverse reactions of antihypertensive drugs in Spain. Part I of the RAAE Study. Blood Press Suppl. 1997;1:11-6.

7. Caro JJ, Salas M, Speckman JL, Raggio G, Jackson JD. Persistence with treatment for hypertension in actual practice. CMAJ. 1999;160:31-7.

8. Pruijm MT, Maillard MP, Burnier M. Patient adherence and the choice of antihypertensive drugs: focus on lercanidipine. Vasc Health Risk Manag. 2008;4(6):1159-66.

9. Messerli FH, Feng Z. Vasodilatory edema: synergistic effect of high-dose calcium antagonist/ACE inhibitor combination therapy (Abstract). Am J Hypertens. 1999;12:121A.

10. Leonetti G, Magnani B, Pessina AC, Rappelli A, Trimarco B, Zanchetti A. COHORT study group. Tolerability of long-term treatment with lercanidipine versus amlodipine and lacidipine in elderly hypertensives. Am J Hypertens. 2002;15(11):932-40.

11. Messerli FH, Grossman E. Pedal edema not all dihydropyridine calcium antagonists are created equal. Am J Hypertens. 2002;15(11):1019-20.

12. Pedrinelli R, Dell'Omo G, Mariani M. Calcium channel blockers, postural vasoconstriction and dependent oedema in essential hypertension. J Hum Hypertens. 2001;15:455-61.

13. Sabbatini M, Leonardi A, Testa R, Vitaioli L, Amenta F. Effect of calcium antagonists on glomerular arterioles in spontaneously hypertensive rats. Hypertension. 2000;35:775-9.

14. Fogari R, Mugellini A, Zoppi A, Corradi L, Rinaldi A, Derosa G, et al. Differential effects of lercanidipine and nifedipine GITS on plasma norepinephrine in chronic treatment of hypertension. Am J Hypertens. 2003;16:596-9.

15. Romito R, Pansini MI, Perticone F, Antonelli G, Pitzalis M, Rizzon P. Comparative effect of lercanidipine, felodipine, and nifedipine GITS on blood pressure and heart rate in patients with mild to moderate arterial hypertension: the Lercanidipine in Adults (LEAD) Study. J Clin Hypertens (Greenwich). 2003;5(4):249-53.

16. Borghi C, Prandin MG, Dormi A, Ambrosioni E. Study group of the regional Unit of the Italian Society of Hypertension. Improved Tolerability of the dihydropyridine calcium-channel antagonist lercanidipine: the lercanidipine challenge trial. Blood Press Suppl. 2003;1:14-21.

17. Barrios V, Navarro A, Esteras A, Luque M, Romero J, Tamargo J, et al. Antihypertensive efficacy and tolerability of lercanidipine in daily clinical practice. The ELYPSE Study. Eficacia de Lercanidipinoy su Perfil de Seguridad. Blood Press. 2002;11:95-100.

18. Barrios V, Escobar C, Navarro A, Calderón A, Ruilope LM. Antihypertensive effectiveness of lercanidipine administered using an electronic pillbox compared with usual care in a cohort of mild-tomoderately hypertensive patients: the ELECTRA study. Therapy. 2007;4(4):433-40.

19. Floras JS. Antihypertensive treatment, myocardial infarction and nocturnal myocardial ischaemia. Lancet. 1988;2:994-6.

Cite this article as: Raparti GT, Choure BK, Patil PT, Patne SS. A randomized comparison between lercanidipine and amlodipine for efficacy and tolerability in patients with essential hypertension. Int J Basic Clin Pharmacol 2016;5:1181-6. 